\title{
Clinical Study Anchor
}

National Cancer Institute

\section{Source}

National Cancer Institute. Clinical Study Anchor. NCI Thesaurus. Code C142436.

A planned activity within a clinical study that marks a transition between epochs or elements within the clinical study. 